\title{
Limiting factors for electron beam lithography when using ultra-thin hydrogen silsesquioxane layers
}

\author{
Anda E. Grigorescu \\ Delft University of Technology \\ Charged Particle Optics Group \\ Delft 2628 CJ, The Netherlands \\ E-mail: A.E.Grigorescu@tudelft.nl
}

Marco C. van der Krogt
Delft University of Technology
Kavli Institute of Nanoscience
Nanofacility, The Netherlands

Cornelis W. Hagen

Delft University of Technology

Charged Particle Optics Group

Delft 2628 CJ, The Netherlands

\begin{abstract}
Isolated dots and lines with $6 \mathrm{~nm}$ width are written in 20-nm-thick hydrogen silsesquioxane (HSQ) layers on silicon substrates, using 100-keV electron beam lithography. The main factors that might limit the resolution, i.e., beam size, writing strategy, resist material, electron dose, and development process, are discussed. We demonstrate that, by adjusting the development process, a very high resolution can be obtained. We report the achievement of $7 \mathrm{~nm}$ lines at a $20-\mathrm{nm}$ pitch written in a 10-nm-thick HSQ layer, using a potassium-hydroxide $(\mathrm{KOH})$ based developer instead of a classical tetra-methyl-ammonium hydroxide (TMAH) developer. This is the smallest pitch achieved to date using $\mathrm{HSQ}$ resist. We think that the resolution can be improved further, and is presently limited by either the beam diameter (which was not measured separately) or by the not-fully-optimized development process. (๑) 2007 Society of Photo-Optical Instrumentation Engineers. [DOI: 10.1117/1.2816459]
\end{abstract}

Subject terms: high resolution; electron beam resist; hydrogen silsesquioxane; electron beam nanolithography; development process.

Paper 07018RR received Feb. 12, 2007; revised manuscript received Jun. 16, 2007; accepted for publication Jul. 3, 2007; published online Dec. 7, 2007. This paper is a revision of a paper presented at the SPIE conference on Advances in Resist Materials and Processing Technology XXIV, Feb. 2007, San Jose, Calif. The paper presented there appears (unrefereed) in SPIE Proceedings Vol. 6519.

\section{Introduction}

When writing nanostructures with electron beam lithography (EBL), at least three main elements are needed. First, a tool must be available that provides a writing beam that is smaller in diameter than the minimum feature dimensions desired. EBL is a promising technique, since an electron beam can easily be focused to a spot of $4 \mathrm{~nm}$ or less. ${ }^{1}$ The writing strategy and the applied area dose also have an important effect on the ultimate resolution that can be achieved with EBL. The second element in nanostructures fabrication is the so-called resist, the medium onto which the desired pattern is written by the electron beam. For nanolithography, where higher acceleration voltages and thinner resists are used, secondary-electron generation close to the incident electron beam is probably the resolution limiting factor, rather than the secondary electrons generated by the backscattered electrons. During e-beam irradiation, either the polymeric structure is cross-linked to form insoluble material (negative tone), or the polymer molecules are broken into smaller soluble molecular fragments (positive tone). In both situations, the smallest definable pattern is at least as large as the molecular area of the polymer molecule on the substrate (several nanometers for most polymeric resists). It is therefore desirable that the area of the resist molecules on the surface is as small as possible. Furthermore, the resist must have a high sensitivity, good resistance to chemical etching, and a good adhesion to the substrate. In the last decade, hydrogen silsesqui-

$1537-1646 / 2007 / \$ 25.00$ @ 2007 SPIE oxane (HSQ, Flowable Oxide, FOx-12 from Dow Corning, Midland, Michigan) became a serious candidate as a high resolution electron beam resist because of its small line edge roughness, high etching resistance, and small molecular size. ${ }^{2}$ HSQ is also an excellent resist for testing e-beam machine resolution limits, because HSQ lines on silicon can be imaged directly in a SEM without the need of gold evaporation for conduction or "lift-off" techniques. In a previous work, ${ }^{3}$ we reported the achievement of 7-nm linewidth at a pitch of $30 \mathrm{~nm}$ written in a 10-nm-thick HSQ layer. The sample was developed for $30 \mathrm{~s}$ using a $\mathrm{KOH}-$ based developer (AZ 400K from Clariant, Somerville, New Jersey) instead of the conventional tetra-methyl-ammonium hydroxide (TMAH). We were also able to achieve 10-nm lines and spaces on a 10-nm-thick HSQ layer. The lines were relatively smooth, which is a major attribute of HSQ as a resist. It was noted that by better optimization of the development process, the resolution could probably be improved further.

The development process is the third important element. A lot of experiments have been done by many workers, which proves the importance of the development time and developer concentration ${ }^{4,5}$ on the resolution. However, to reach sub-10-nm resolution, a variety of extra process steps, ${ }^{6,7}$ before, during, or after electron beam irradiation, have often been necessary.

This current work is focused on experiments with ultrathin $(10$ and $20 \mathrm{~nm})$ resist layers. All the experiments reported in the HSQ literature were made using 50- to $100-\mathrm{nm}$ resist layers and by using the standard TMAH-based developer. We believe that thinner resist lay- 
Grigorescu, van der Krogt, and Hagen: Limiting factors for electron beam lithography...

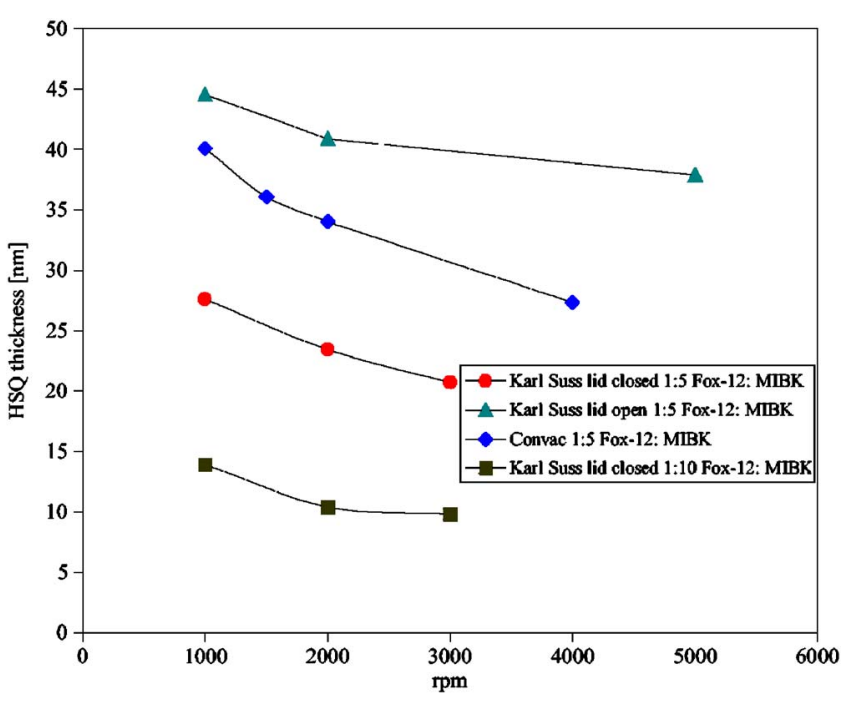

Fig. 1 Spin curve for HSQ at different dilution rates and at different rotation speeds, when using Karl Suss spinner (with the lid closed or open) and a Convac spinner.

ers and a better optimization of the development process may improve the resolution that can be achieved with HSQ as a high resolution electron beam resist. It is the objective of this work to investigate the important factors that may limit the ultimate resolution that can be achieved with HSQ by writing small dots, lines, and spaces.

\section{Experiment}

For the e-beam exposure experiments, we used $19 \times 19-\mathrm{mm}$ samples diced from (100)-oriented, 20 to $30 \Omega \mathrm{cm}$, p-type (B-doped) silicon 4-in. wafers. First, the samples were cleaned with ultrasonic agitation in $100 \%$ fuming nitric acid, demineralized water, and isopropanol for $2 \mathrm{~min}$, and finally blown dry with nitrogen gas. Next, the samples were baked at $200{ }^{\circ} \mathrm{C}$ for $2 \mathrm{~min}$ to remove residual moisture. For high resolution patterning, the silicon wafers were spin coated with a solution of 1:5 FOx-12: methyl isobutyl ketone (MIBK) or 1:10 FOx-12: MIBK at different rotation speeds varying from 1000 to $5000 \mathrm{rpm}$ for $60 \mathrm{~s}$. To get the thinnest possible resist layers, two different spinners were used. On a Karl Suss (Garching, Germany) spinner, two spinning sessions were made: one with the lid closed (to decrease the rate of evaporation of the solvent) and one with the lid open. From Fig. 1 we can see that an approximately 20-nm-thick HSQ layer is obtained, when a solution of 1:5 FOx-12: MIBK is spun at $3000 \mathrm{rpm}$ for $60 \mathrm{~s}$, using the Karl Suss spinner with closed lid. When the lid is open, almost a double thickness $(40 \mathrm{~nm})$ is obtained. For comparison, the same experiment was done on a Convac (Vaihingen, Germany) spinner, and the result was a 32-nm-thick HSQ resist layer. By using a higher dilution rate 1:10 FOx-12: MIBK, a 10-nm-thick HSQ layer was obtained at $3000 \mathrm{rpm}$ for $60 \mathrm{~s}$ on the Karl Suss spinner with the lid closed. The HSQ spin coating was performed directly on the silicon wafer without using a primer. After spinning, the HSQ layer was prebaked on a hotplate for $40 \mathrm{~min}$ at $90{ }^{\circ} \mathrm{C}$ to get a high contrast and good reproducibility. ${ }^{4}$ The thickness of the resulting HSQ, rang-

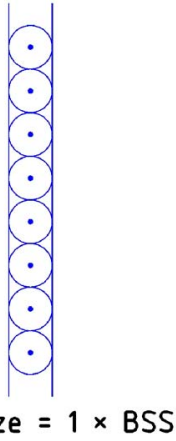

(a)

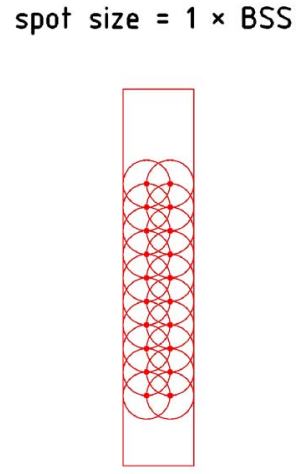

spot size $=0.5 \times$ BSS

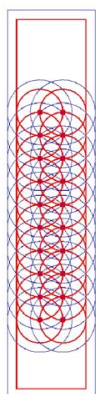

inner circles: spot size $=2 \times$ BSS spot size $=2 \times$ BSS outer circles: higher dose

(b)

Fig. 2 Schematic representation of the possible writing strategies: (a) single exel, single pass: designed linewidth=BSS, and (b) $n$ exel, single pass: designed linewidth $=n \times \mathrm{BSS}, n=2$ in the case shown.

ing from 10 to $20 \mathrm{~nm}$, was measured with a Gaertner (Skokie, Illinois) ellipsometer. The wafers were exposed in a Vistec (Best, The Netherlands) Electron Beam Pattern Generator (EBPG 5000+) at $100 \mathrm{kV}$ with an aperture of $300 \mu \mathrm{m}$ (201-pA beam current, 2-nm estimated spot size). The test pattern consists of five sets of $4-\mu \mathrm{m}$-long lines. Within each set, all lines are designed with a fixed width and pitch, but between the sets the linewidth and pitch varies. Chosen widths are $1.25,2.5,5,7.5$, and $10 \mathrm{~nm}$ at a pitch of $50,75,100,120$, and $150 \mathrm{~nm}$, respectively. The structures were all written with a fixed beam step size (BSS, the distance between two adjacent exposures) of $1.25 \mathrm{~nm}$. Depending on the designed linewidth, the exposure of a line is performed by scanning the beam once (single pass) over either one line (single exel or 1-exel) or $n$ adjacent lines ( $n$-exel line). The number of exels is defined as the number of times the BSS fits into the designed linewidth. A schematic representation of the writing strategy is illustrated in Fig. 2.

In principle, the smaller the beam diameter that is used, the thinner the resulting line can become [see Fig. 2(a)]. However, if the beam diameter is, for example, equal to half the BSS, the line obtained after developing is not continuous. In Fig. 2(b), an example of a line is shown for which the designed width is two times the BSS. The beam diameter is two times as large as the BSS. When the same line is written at a higher dose, the linewidth is broadened because of the proximity exposure (the outer circles). However, to write the thinnest lines possible, single exel lines can be written, where the linewidth is equal to the beam 
size. Of course the linewidth also depends on the exposure dose. By doing a dose test, an optimum dose can be determined for which the linewidth after exposure and development is equal to the designed linewidth. For simplicity, and because the beam size is not accurately known, we define the designed linewidth as the BSS multiplied by the number of exels. The line dose is calculated by multiplying the area dose with the designed linewidth. The design mentioned before was written in an array of $10 \times 10$ cells, each cell containing five sets of lines. Each cell was exposed with a different electron dose. The starting dose was $3000 \mu \mathrm{C} / \mathrm{cm}^{2}$ (unless otherwise stated), and the dose of each next cell is found by multiplication with a factor of 1.048 , leading to an end dose of $311092 \mu \mathrm{C} / \mathrm{cm}^{2}$. We also exposed dots by writing a design with an array of the smallest possible pattern: squares measuring $1.25 \times 1.25 \mathrm{~nm}$ $(1 \times 1 \mathrm{BSS})$, each representing a single, isolated exposure. The pitch was $125 \mathrm{~nm}$ in the $x$ direction and $100 \mathrm{~nm}$ in the $y$ direction. A dose test was performed by writing this design in an array of $10 \times 10$ cells, each cell exposed with a different area dose. The starting dose was $5000 \mu \mathrm{C} / \mathrm{cm}^{2}$ (unless otherwise stated) and the dose of each following cell is found by multiplication with a factor of 1.055 , leading to an end dose of $1 \mathrm{C} / \mathrm{cm}^{2}$. The samples were developed by manual immersion at $20{ }^{\circ} \mathrm{C}$ in TMAH based developer (MF-322 from Rohm and Haas, Philadelphia, Pennsylvania) for $60 \mathrm{~s}$, unless otherwise stated. Exposed substrates were then rinsed in 1:9 developer: demineralized water for $10 \mathrm{~s}$, rinsed in demineralized water for another $10 \mathrm{~s}$, and blown dry with nitrogen. Following the development process, the resist linewidth was imaged using an FEI (Eindhoven, The Netherlands) Nova Nano 200 scanning electron microscope (SEM). A number of line scans (intensity as function of distance) was integrated using the Digital Micrograph program(Gatan, Inv., Pleasanton, California). ${ }^{3}$ The full width at half maximum value of a line in this integrated intensity profile is considered to be the measured linewidth. Dots were measured using the same method as described before.

\section{Results and Discussion}

\subsection{Isolated Features: Dots}

First, we describe the resolution obtained for nanostructures that we call "isolated," meaning that the pitch between adjacent features is much larger than the size of the designed features. Experiments on resolution limits were conducted on single layers of HSQ of 10 and $20 \mathrm{~nm}$ thick by exposure of test patterns consisting of dots and lines with different widths and pitches written with various exposure doses. Dots were realized by writing a design with the smallest possible pattern: squares measuring $1.25 \times 1.25 \mathrm{~nm}(1 \times 1$ BSS), each representing a single, isolated exposure. Using $100-\mathrm{keV}$ electron beam lithography, we report the achievement of 6-nm dots (see Fig. 3) with a pitch of $125 \mathrm{~nm}$ in the $x$ direction and $100 \mathrm{~nm}$ in the $y$ direction in a $20-\mathrm{nm}$ HSQ layer on silicon substrates. Although the pitch is relatively large, this result is important because these are the smallest dots ever written in HSQ. The sample was developed for $60 \mathrm{~s}$ using MF-322 developer. Figure 4 shows the results of dot size as function of the area dose. The starting dose was $5000 \mu \mathrm{C} / \mathrm{cm}^{2}$ and the dose of each following cell

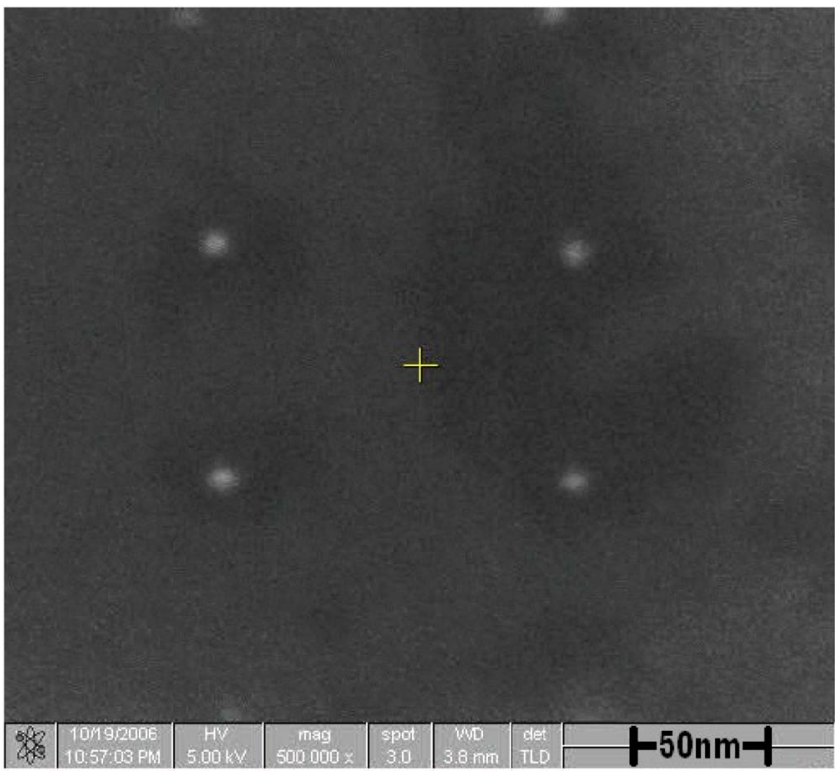

Fig. 3 SEM image of 6.3-nm dots (with 125-nm pitch in the $x$ direction and $100-\mathrm{nm}$ pitch in the $y$ direction) written in a $20-\mathrm{nm}$ HSQ layer at $100 \mathrm{keV}$. The area dose was $556143 \mu \mathrm{C} / \mathrm{cm}^{2}$.

is found by multiplication with a factor of 1.055 , leading to a final dose of $1 \mathrm{C} / \mathrm{cm}^{2}$. It is seen that the dot size increases with the area dose. From the SEM inspection, we observed that the first cell, where a regular array of dots appears, is found at a very high dose, i.e., $527150 \mu \mathrm{C} / \mathrm{cm}^{2}$. The first dots are visible at a dose of $212150 \mu \mathrm{C} / \mathrm{cm}^{2}$, but the array is not complete. Below this dose, the structures are not visible at all. We expected the regular array of dots to be visible at a lower dose. The fact that the written structures start to be visible only at doses higher than $212150 \mu \mathrm{C} / \mathrm{cm}^{2}$ may be simply due to the fact that the electron dose range was chosen to start at too small values. But it could also be that the development time was too long for these thin layers, such that the structures were washed away. It is noted here that most of the experiments reported in the HSQ literature using TMAH-based developer and development times of $60 \mathrm{~s}$ were done on much thicker re-

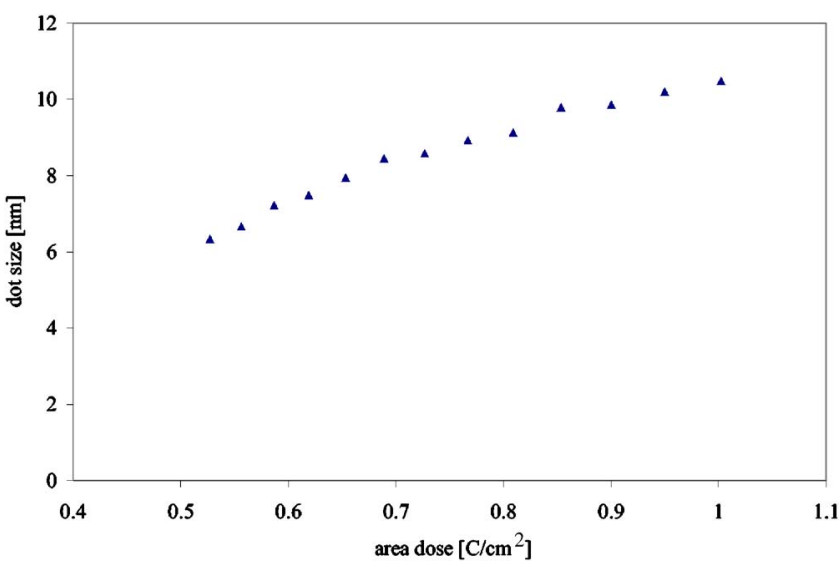

Fig. 4 Dot size as function of the area dose written in 20-nm-thick HSQ. 
Grigorescu, van der Krogt, and Hagen: Limiting factors for electron beam lithography...

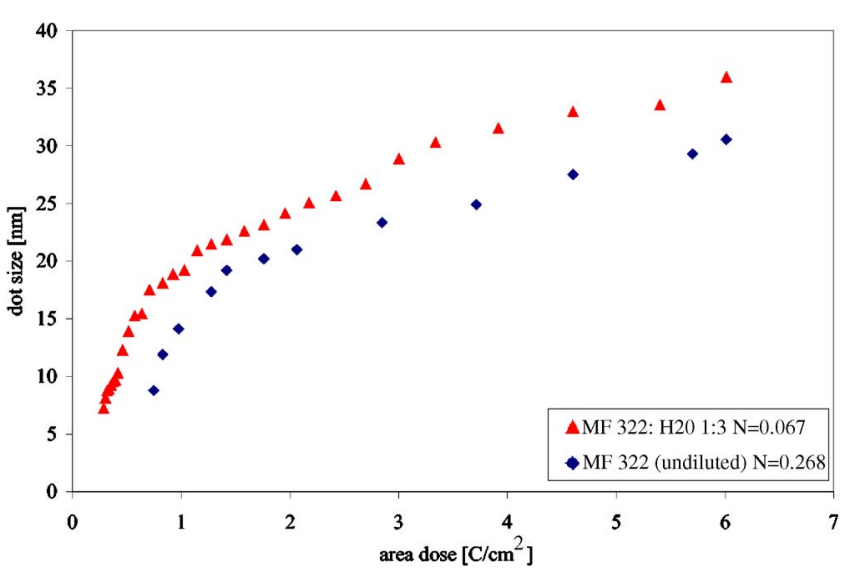

Fig. 5 Dot size as a function of the area dose written in 20-nm-thick HSQ, when two different concentrations for TMAH are used: $N$ $=0.067$ (triangles) and $\mathrm{N}=0.268$ (squares).

sist layers typically 50 to $400 \mathrm{~nm}$ thick. For our relatively thin layers, a shorter development time might be sufficient and also a smaller feature size is to be expected. Of course, it cannot be ruled out that the beam size is still limiting the resolution, as it was not measured separately.

To quantify the influence of the development process on the feature size, one can either change the development time or the strength (normality) of the developer. We performed experiments in which the whole process was identical, except for the development process. In the first set of experiments, we changed the development time from $60 \mathrm{~s}$ to 45 and $30 \mathrm{~s}$, respectively. The results of these experiments do not show a significant improvement of the resolution. Although it is possible that even shorter development times do improve the resolution, we decided to change the strength of the developer instead.

In this second set of experiments, we kept the development time constant $(60 \mathrm{~s})$, but we varied the strength of the developer. In Fig. 5, the dot size as a function of the area dose is indicated for two different concentrations of TMAH developer. MF-322 was used undiluted $(\mathrm{N}=0.268 \mathrm{eq} / \mathrm{l})$ and diluted with demineralized water in a ratio $1: 3$ $(\mathrm{N}=0.067 \mathrm{eq} / 1)$. For diluted developer, the regular arrays of dots appear at a lower electron dose than when the developer is undiluted. Since all samples are exposed with the same dose range, this is a clear indication that the structures that turn out to be well developed with the diluted developer are washed away in the undiluted case. Still, no better resolution than $6 \mathrm{~nm}$ was obtained.

In a third set of experiments, we changed the developer type by using a potassium hydroxide $(\mathrm{KOH})$ aqueous solution instead of TMAH. Namatsu et al. ${ }^{7}$ suggested that this novel developer could be used when high resolution is required. One sample was developed with TMAH (N $=0.268 \mathrm{eq} / 1)$ for $60 \mathrm{~s}$, and the other one was developed for $10 \mathrm{~s}$ with a $\mathrm{KOH}$ aqueous solution $(\mathrm{N}=1.39 \mathrm{eq} / \mathrm{l})$ (see Fig. $6)$. Although in terms of ultimate resolution we obtained the same results (almost 8-nm dots for both samples), an interesting effect occurs when the sample is developed with AZ $400 \mathrm{~K}$ from Clariant. Even for a six-times-shorter development time, the features are washed away when a stronger

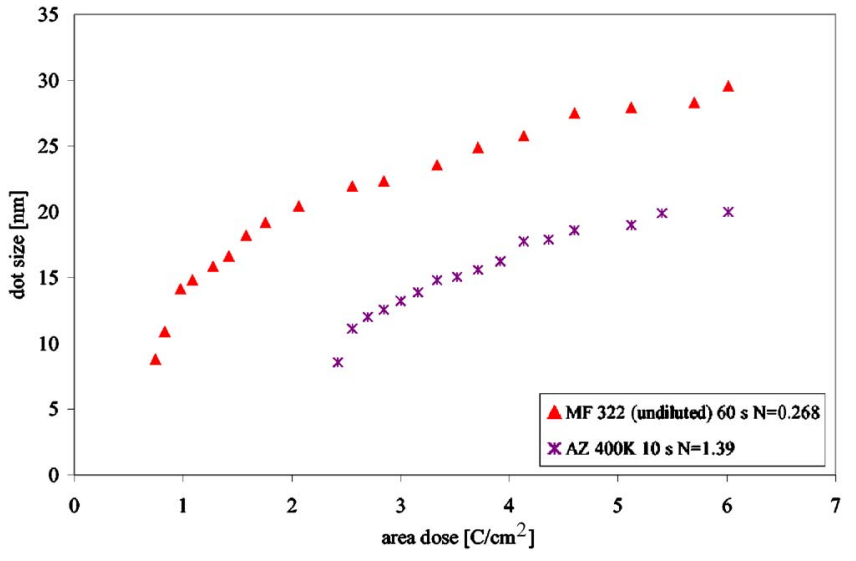

Fig. 6 Dot size as function of the area dose written in 20-nm-thick $\mathrm{HSQ}$, when two different developers are used: TMAH, $60 \mathrm{~s}$; and $A Z$ 400K, $10 \mathrm{~s}$.

developer (higher normality) is used. The first visible regular array of dots needs a three-times-higher dose when the sample is developed with AZ 400K compared to MF-322.

In terms of ultimate resolution, we obtained a minimum dot size of approximately $6 \mathrm{~nm}$ with a pitch of $125 \mathrm{~nm}$ in the $x$ direction and $100 \mathrm{~nm}$ in the $y$ direction. This resolution may be limited by the beam diameter, which was not measured separately, or by a still not well optimized development process.

\subsection{Isolated Features: Isolated Lines}

We also wrote lines with different widths and pitches at various exposure doses. The lines were all written with a fixed BSS of $1.25 \mathrm{~nm}$. Depending on the designed linewidth, an exposure is performed by scanning the beam once (single pass) over either one (single exel or 1-exel) or $n$ adjacent lines ( $n$-exel line). The sample was developed for $60 \mathrm{~s}$ using MF-322. The line dose is calculated by multiplying the area dose with the designed linewidth. The line dose is a convenient parameter when comparing lines with different designed width. For example, with a constant line dose, a higher exel line at a lower area dose can give the same result as a lower exel line at a higher area dose, because of proximity exposure and overlap of adjacent exels. ${ }^{3}$ When the SEM inspection is performed, the lines that are visible at the lowest area doses are the 8-exel lines (10-nm designed linewidth). This is to be expected, since the line dose is the highest for these lines. The same general behavior is observed as for the dots: if the dose increases, the lines become wider (Fig. 7). For doses of $3453 \mu \mathrm{C} / \mathrm{cm}^{2}$ and $4365 \mu \mathrm{C} / \mathrm{cm}^{2}$, the measured linewidths (after development) are 5.5 and $8.7 \mathrm{~nm}$, respectively [see Figs. 7(a) and 7(b)], which are smaller than the designed linewidth of $10 \mathrm{~nm}$. This may be due to the fact that the dose was too low and the structures were not completely exposed. At a dose of $5518 \mu \mathrm{C} / \mathrm{cm}^{2}$, we measured 10.5 -nm linewidth, and this can be called the optimum dose because the measured linewidth after development is equal to the designed linewidth [Fig. 7(c)]. For higher doses, i.e., $49980 \mu \mathrm{C} / \mathrm{cm}^{2}, 40-\mathrm{nm}$ lines are obtained [Fig. 7(d)]. In this case, the structure is clearly overexposed. The thinnest 6- and 4-exel lines (7.5- and 5-nm designed linewidth) 


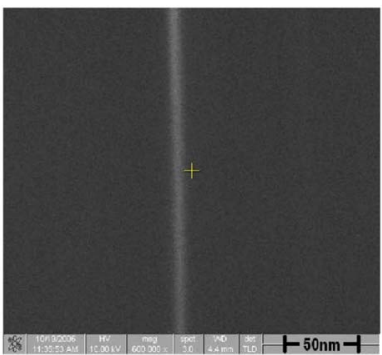

(a)

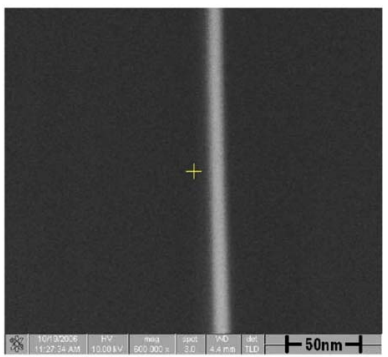

(c)

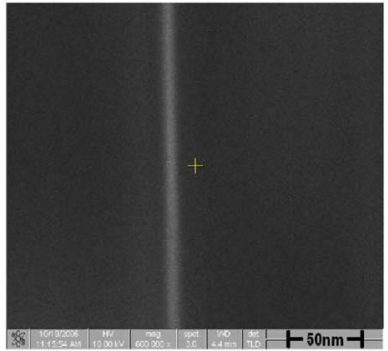

(b)

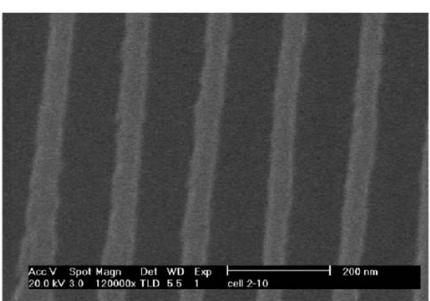

(d)
Fig. 7 Sequence of SEM micrographs, showing 10-nm designed lines (8-exel, single pass exposure) exposed at $100 \mathrm{keV}$, with four different doses at a pitch of $150 \mathrm{~nm}$. The sample was developed for $60 \mathrm{~s}$ with TMAH. (a) Area dose of $3453 \mu \mathrm{C} / \mathrm{cm}^{2}$ (line dose: $3.45 \mathrm{nC} / \mathrm{cm}$ ); measured linewidth is $5.5 \mathrm{~nm}$. (b) Area dose of $4365 \mu \mathrm{C} / \mathrm{cm}^{2}$ (line dose: $4.37 \mathrm{nC} / \mathrm{cm}$ ); measured linewidth is $8.7 \mathrm{~nm}$. (c) Area dose of $5518 \mu \mathrm{C} / \mathrm{cm}^{2}$ (line dose: $5.52 \mathrm{nC} / \mathrm{cm}$ ); measured linewidth is $10.5 \mathrm{~nm}$. (d) Area dose of $49980 \mu \mathrm{C} / \mathrm{cm}^{2}$ (line dose: $50.0 \mathrm{nC} / \mathrm{cm}$ ); measured linewidth is $40 \mathrm{~nm}$.

(Fig. 8) are found at a dose of $5266 \mu \mathrm{C} / \mathrm{cm}^{2}$ [for 6-exel, see Fig. 8(a)] and $7662 \mu \mathrm{C} / \mathrm{cm}^{2}$ [for 4-exel, see Fig. 8(b)]. Measured linewidths are 5.7 and $5.5 \mathrm{~nm}$, respectively. For the 6-exel line, this dose appears to be below the optimum dose, since the measured width $(5.7 \mathrm{~nm})$ is smaller than the designed value of $7.5 \mathrm{~nm}$. For the 4-exel line, the measured and designed linewidth are in close agreement. Figures 8(a) and 8(b) also show the usefulness of the line dose: with a different number of exels (4 versus 6) and different area doses $\left(7662\right.$ versus $\left.5266 \mu \mathrm{C} / \mathrm{cm}^{2}\right)$, the line doses $(3.83$ and $3.95 \mathrm{nC} / \mathrm{cm})$ and measured linewidths $(5.5$ and $5.7 \mathrm{~nm})$ are comparable. The 2- and 1-exel lines (2.5- and 1.25-nm designed linewidth) appear at the highest doses: 17818 and $27171 \mu \mathrm{C} / \mathrm{cm}^{2}$, respectively [Figs. 8(c) and 8(d)]. The measured linewidth is almost six (for 1-exel) and four times (for 2-exel) larger than the designed linewidth, so it appears that the structures were overexposed. This could also be explained by a too long development time, such that the structures written at lower doses were washed away. Other explanations might be that the beam size is larger than the designed linewidth, or that the limit in resolution of the HSQ resist is reached.

\subsection{Dense Features}

The real resolution test in lithography is, of course, the fabrication of dense lines and spaces. In a previous paper, ${ }^{3}$ we reported the achievement of 7-nm linewidth at a pitch of $30 \mathrm{~nm}$ written in a 10-nm-thick HSQ layer. The sample was developed for $30 \mathrm{~s}$ using a $\mathrm{KOH}$-based developer instead of the conventional TMAH. Next, we were able to

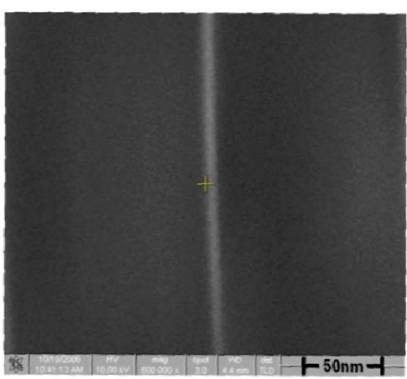

(a)

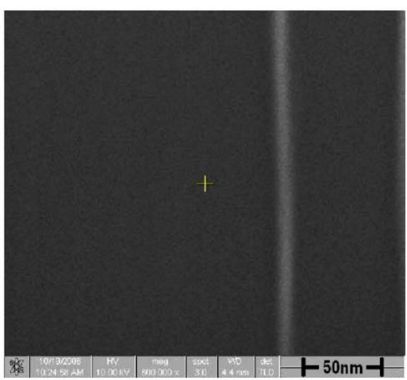

(c)

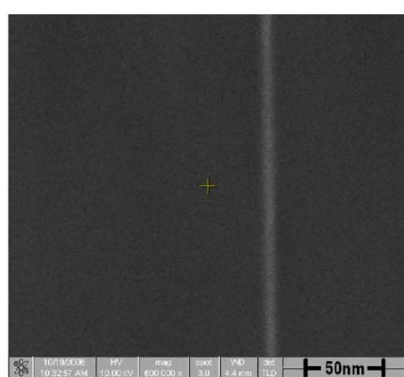

(b)

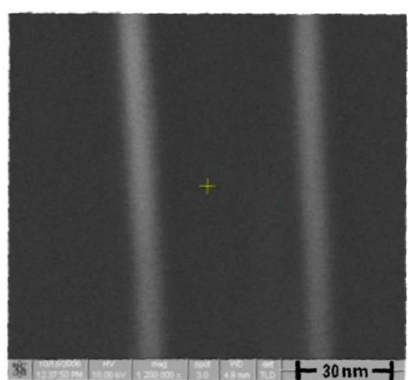

(d)
Fig. 8 SEM micrographs of $n$-exel, single pass exposures (1.25-nm beam step size, 100-keV beam energy) written in a 20-nm-thick HSQ layer at four different area doses. (a) 6-exel, single pass exposure with an area dose of $5266 \mu \mathrm{C} / \mathrm{cm}^{2}$ (line dose: $3.95 \mathrm{nC} / \mathrm{cm}$ ); measured linewidth is $5.7 \mathrm{~nm}$ and the pitch is $120 \mathrm{~nm}$. (b) 4-exel, single pass exposure with an area dose of $7662 \mu \mathrm{C} / \mathrm{cm}^{2}$ (line dose: $3.83 \mathrm{nC} / \mathrm{cm}$ ); measured linewidth is $5.5 \mathrm{~nm}$ and the pitch is $100 \mathrm{~nm}$. (c) 2-exel, single pass exposure with an area dose of $17818 \mu \mathrm{C} / \mathrm{cm}^{2}$ (line dose: $4.45 \mathrm{nC} / \mathrm{cm}$ ); measured linewidth is $10.4 \mathrm{~nm}$ and the pitch is $75 \mathrm{~nm}$. (d) 1-exel, single pass exposure with an area dose of $27171 \mu \mathrm{C} / \mathrm{cm}^{2}$ (line dose: $3.39 \mathrm{nC} / \mathrm{cm}$ ); measured linewidth is $7.8 \mathrm{~nm}$ and the pitch is $50 \mathrm{~nm}$.

achieve 10-nm lines and spaces on a 10-nm-thick HSQ layer. The lines were relatively smooth, which is a major attribute of HSQ as a resist.

To see whether the resolution could be further improved, a new experiment was conducted on a 10-nm-thick HSQ layer with the same design as described in Ref. 3 (lines with $20 \mathrm{~nm}$ pitch). Again, this design was written in an array of $10 \times 10$ cells, each of them exposed with a different electron dose. The starting dose was $750 \mu \mathrm{C} / \mathrm{cm}^{2}$, and the dose of each following cell is found by multiplication with a factor of 1.048, leading to an end dose of $77773 \mu \mathrm{C} / \mathrm{cm}^{2}$. These doses are four times smaller than used for the isolated lines, described earlier, because we wanted to diminish the proximity effect that can occur when dense arrays of structures are written. The sample was developed for $15 \mathrm{~s}$ using AZ 400K. The reason for choosing this relatively short development time was to prevent the structures from being washed away, since this $\mathrm{KOH}$-based developer is almost five times stronger than MF-322. The smallest lines obtained in a 10-nm-thick HSQ layer are shown in Fig. 9. In Fig. 9(a), 7-nm-wide lines at a pitch of $20 \mathrm{~nm}$ are shown resulting from a 2-exel (i.e., 2.5-nm designed width) single pass exposure with an area dose of $70812 \mu \mathrm{C} / \mathrm{cm}^{2}$. This is a clear improvement of the result obtained before. ${ }^{3}$ In Fig. 9(b), 4-exel (i.e., $5 \mathrm{~nm}$-designed width) single pass lines with area dose of $36732 \mu \mathrm{C} / \mathrm{cm}^{2}$ are shown, which have a measured width 


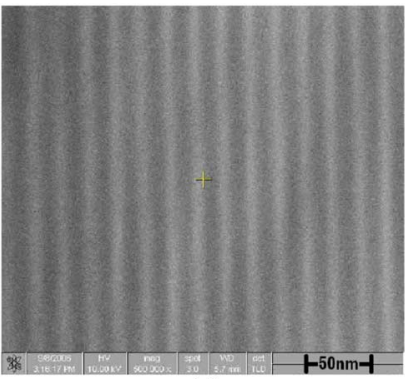

(a)

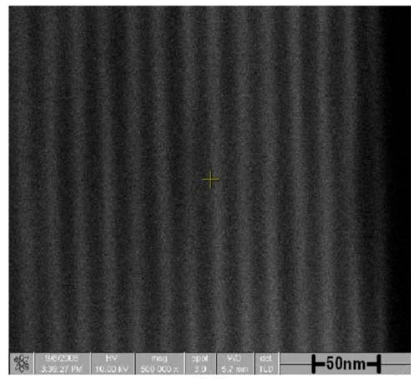

(b)
Fig. 9 SEM micrographs of $n$-exel, single pass exposures (1.25-nm beam step size, 100-keV beam energy) written in a 10-nm HSQ layer at two different area doses. The pitch is $20 \mathrm{~nm}$. (a) 2-exel, single pass exposure with an area dose of $70812 \mu \mathrm{C} / \mathrm{cm}^{2}$ (line dose: $17.7 \mathrm{nC} / \mathrm{cm}$ ); measured linewidth is $7 \mathrm{~nm}$. (b) 4-exel, single pass exposure with an area dose of $36732 \mu \mathrm{C} / \mathrm{cm}^{2}$ (line dose: $18.4 \mathrm{nC} / \mathrm{cm})$; measured linewidth is $7.5 \mathrm{~nm}$.

of $7.5 \mathrm{~nm}$. Looking at the dose, we see that the $2.5-\mathrm{nm}$ designed linewidth needs almost a double dose to be completely developed, in comparison with the 5-nm designed linewidth. This may be caused by the proximity effect, which contributes to the final linewidth.

\section{Conclusions}

We show that ultra-thin layers (10 to $20 \mathrm{~nm}$ ) of HSQ can be obtained when a spinner is used that has a small volume between lid and spinning chuck (Karl Suss spinner with lid closed compared to a Convac spinner). In terms of ultimate resolution, we obtain $6-\mathrm{nm}$ isolated dots. The dot size increases approximately from 6 to $10 \mathrm{~nm}$ when increasing the area dose from 0.5 to $1 \mathrm{C} / \mathrm{cm}^{2}$. The dot size is not influenced much by decreasing the development time from 60 to $30 \mathrm{~s}$. However, by diluting the developer, while keeping the exposure dose constant, the dot size increases. Furthermore, the lowest dose that results in well-developed dots shifts to lower values. This suggests that small features exposed at small doses are washed away when too strong developers are used. The same behavior is observed when comparing a $\mathrm{KOH}$-based developer (strong) with TMAH (weaker).

The fact that when using dilute developer solutions, the dots at low exposure doses are not significantly smaller than the smallest dots developed in strong developers, suggests that the dot size is probably limited by the electron probe size. Using TMAH as a developer, 5.5-nm-wide isolated lines are obtained in a 20-nm-thick HSQ layer with a 4-exel, single pass, exposure at $7662 \mu \mathrm{C} / \mathrm{cm}^{2}$. By refining the development process (shorter development time, a novel developer), 7-nm lines at a 20 -nm pitch were written in a 10-nm-thick HSQ layer, using $100 \mathrm{keV}$, 2-exel single pass, e-beam exposure at a dose of $70812 \mu \mathrm{C} / \mathrm{cm}^{2}$. This represents the smallest pitch achieved to date using HSQ as electron beam resist.

In principle, when writing sub-10-nm structures, the resolution is influenced by multiple factors such as beam size, writing strategy, area dose, resist thickness, and the development process. By varying the writing strategy (number of exels), the area dose, and the development process, we demonstrate that resolution improvements could still be achieved. Further work is needed to quantify all these parameters and to find the ideal recipe for the ultimate resolution that can be achieved with HSQ electron beam resist. At present, we cannot conclude yet whether the resolution is limited either by the beam size (which is not measured separately) or by the development process. We plan to write dense lines and spaces in a scanning transmission electron microscope (STEM), which has a very small probe size of $0.3 \mathrm{~nm}$. When the probe size is still limiting the resolution, this will clearly lead to better results.

\section{References}

1. K. Yamazaki and H. Namatsu, "5-nm-order electron-beam lithography for nanodevice fabrication," Jpn. J. Appl. Phys., Part 1 43, 37673771 (2004)

2. H. Namatsu, Y. Takahashi, K. Yamazaki, M. Nagase, and K. Kurihara, "Three-dimensional siloxane resist for the formation of nanopatterns with minimum linewidth fluctuations," J. Vac. Sci. Technol. B 16, 69-76 (1998).

3. A. E. Grigorescu, M. C. van der Krogt, C. W. Hagen, and P. Kruit, "10 nm lines and spaces written in HSQ, using electron beam lithography," Microelectron. Eng. 84, 822-824 (2007).

4. W. Henschel, Y. M. Georgiev, and H. Kurz, "Study of a high contrast process for hydrogen silsesquioxane as a negative tone electron beam resist," J. Vac. Sci. Technol. B 21, 2018-2025 (2003).

5. D. Lauvernier, J. P. Vilcot, M. Francois, and D. Decoster, "Optimization of HSQ resist e-beam processing technique on GaAs material," Microelectron. Eng. 75, 177-182 (2004).

6. M. J. Lercel, H. G. Craighead, A. N. Parikh, K. Seshadri, and D. L. Allara, "Sub-10 nm lithography with self-asembled monolayers," Appl. Phys. Lett. 68, 1504-1506 (1996).

7. H. Namatsu, K. Kurihara, M. Nagase, and T. Makino, "Fabrication of 2-nm-wide silicon quantum wires through a combination of a partially-shifted resist pattern and orientation-dependent etching," Appl. Phys. Lett. 70, 619-621 (1997).

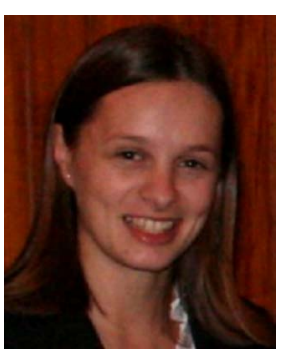

Anda E. Grigorescu is a PhD student working in the charged particle optics group at Delft University of Technology, The Netherlands. Her primary area of interest, at present, is the study of ultimate resolution that can be achieved when using ultra-thin HSQ layers as high resolution electron beam resists. She also developed a Monte Carlo simulation program that calculates not only the trajectories and the energies of the scattered secondary electrons, both in the substrate and resist, but also the bond-breaking distributions in the resist. The aim of the simulation is to optimize the parameters, such as incident energy, substrate material, and resist material, to obtain the ultimate resolution.

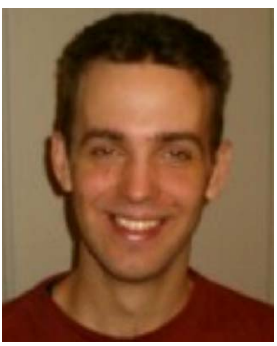

Marco C. van der Krogt received his BSc in engineering physics (major: applied physics) from the Rijswijk Institute of Technology (RITE), after a graduation period at Leiden University. In 2002, he joined the Nanofacility of the Kavli Institute of Nanoscience at Delft University of Technology. He is a specialist in a wide spectrum of nanodevice fabrication techniques. His main topic is thin film processing. 
Grigorescu, van der Krogt, and Hagen: Limiting factors for electron beam lithography...

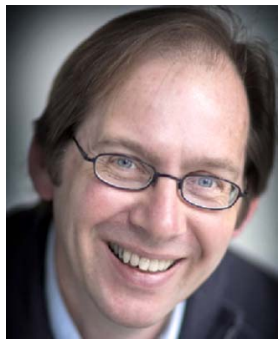

Cornelis W. Hagen has been an assistant professor at Delft University of Technology since 1994. His area of research is microscopy and lithography with charged particles. $\mathrm{He}$ is involved in the development of novel electron and ion sources, and his main interest, at present, is focused electronbeam-induced processing and resist-based

electron beam lithography for the fabrication

of structures with sizes between 1 and

$20 \mathrm{~nm}$. He is a coauthor of more than 30 journal papers and numerous publications in conference proceedings. [Photograph by Jacqueline de Haas.] 